\title{
On classical and quantum liftings
}

\author{
L. Accardi ${ }^{1}$, D. Chruściński ${ }^{2}$, A. Kossakowski ${ }^{2}$, T. Matsuoka ${ }^{3}$ and M. Ohya ${ }^{4}$ \\ ${ }^{1}$ University of Roma Tor Vergata, Roma, Italy \\ 2 Nicolaus Copernicus University, Torun, Poland \\ 3 Tokyo Universiy of Science, Suwa, Japan \\ 4 Tokyo Universiy of Science, Noda, Japan
}

\begin{abstract}
We analyze the procedure of lifting in classical stochastic and quantum systems. It enables one to 'lift' a state of a system into a state of 'system+reservoir'. This procedure is important both in quantum information theory and the theory of open systems. We illustrate the general theory of liftings by a particular class related to so called circulant states.
\end{abstract}

\section{Introduction}

The interest on quantum entanglement has dramatically increased during the last two decades due to the emerging field of quantum information theory [1]. It turns out that quantum entanglement may be used as a basic resource in quantum information processing and communication. The prominent examples are quantum cryptography, quantum teleportation, quantum error correction codes and quantum computation (see the recent review [2]).

The characteristic feature of an entangled state (already stressed by Schrödinger) is that knowing a composed system that is in a pure state one can not say the same thing, in general, for any of its subsystems. Therefore, a full knowledge of a system can guarantee only a partial knowledge of its subsystems. This can't happen in the classical case i.e. it is a purely quantum feature. In this paper we analyze the converse problem: suppose one knows a state of a subsystem. How one can 'lift' this state into a state of the whole system? An appropriate mathematical framework was provided in [3, 4]. This problem is important in many applications of quantum information theory and quantum dynamics.

For example it is of primary importance to be able to construct a state of a composite system knowing only its marginals, i.e. the states of subsystems. An appropriate lifting enables one to define a compound state which takes into account correlations between subsystems [3, 4, 5]. Another important issue arises in the quantum dynamics of open systems [7, 8]. One is interested to extract the dynamics of a quantum system from the unitary dynamics of the 'system + environment'. Due to the presence of system-environment correlations this problem is highly nontrivial. One usually assumes that initially 'system + environment' was described by a product state, i.e. there were no correlations at all. The standard definition of reduced dynamics implies then that the dynamics of the system is completely positive and trace preserving. A product state provides a very special 
example of lifting: a system state $\rho$ is lifted to 'system + environment' state $\rho \otimes \omega$. Could we use other liftings to define initial 'system + environment' state?

One tries to relax the condition upon the 'product lifting' and considers dynamics which is only positive but not completely positive (see e.g. [11, 12, 13, 14]). Such positive dynamics enables one to map density matrices into legitimate density matrices. However, one looses the important property that coupling the system to an arbitrary environment will result in positive 'system + environment' dynamics. This problem appeared more recently in the context of non-Markovian evolution (see recent papers [15, 16]). An interesting debate upon this problem started already in the 90 . of the last century [9, 10].

The problem of positive but not completely positive maps is very much related to the problem of detection of quantum entanglement (see 2] for the review). It is well known that the general structure of positive maps is still unknown and only partial results are available (see [17, 18, 19, 20, 21, 22 for recent examples and constructions). One may try to use appropriate liftings to provide new classes of such maps. Suppose for example that we lift a state $\rho$ to the compound state [3, 4], perform a positive map and then reduce to the system of interest. It is obvious that the resulting map would be positive and it depends very much upon the applied lifting. An interesting open problem is the classification of 'lifting assisted maps' defined this way.

The paper is organized as follows: for pedagogical reason we start with presentation of basis ideas of stochastic classical systems Section 2, In particular we show how to reformulate the classical theory in the quantum framework. Section 3 provides the discussion of classical liftings and Section 4 provides generalization for multipartite case. Then we consider the main topic of this paper in Section 5. Section 6 illustrates the general ideas of liftings by 'circulant liftings'. These are liftings related to the construction of so called circulant states [23]. In particular we analyze Bell diagonal liftings which play important role in quantum information theory (see [25, 26] and recent paper [27]). Final conclusions are collected in the last Section.

\section{The quantum framework for classical probability with finite state space}

\section{$2.1 \quad$ Preliminaries}

Let us consider a classical $n$-level stochastic system. The primary object for elementary classical probability, i.e. probability on a finite state space, is the space of elementary events $\Omega=$ $\left\{\omega_{1}, \ldots, \omega_{n}\right\}$. The corresponding space of states $S(\Omega)$ consists of probability distributions over $\Omega$

$$
S(\Omega):=\left\{p=\left(p_{1}, \ldots, p_{n}\right) \mid p_{i} \geq 0, \sum_{i=1}^{n} p_{i}=1\right\} .
$$

It is clear that $S(\Omega)$ is convex and the corresponding set of extremal points - pure states - reads as follows

$$
S_{0}(\Omega):=\left\{p \in S(\Omega) \mid p_{i}^{2}=p_{i}, i=1, \ldots, n\right\} .
$$

Hence, a state $p \in S(\Omega)$ is pure iff $p_{i}=\delta_{i j}$ for some $j \in\{1, \ldots, n\}$. It is evident that $S_{0}(\Omega)$ consists of the $n$ vertices of $(n-1)$-dimensional simplex $S(\Omega)$. A classical observable is a random variable 
$a: \Omega \longrightarrow \mathbb{R}$, and the corresponding expectation value of $a$ in the state $p \in S(\Omega)$ is given by

$$
\langle a, p\rangle:=\sum_{i=1}^{n} a_{i} p_{i}=\sum_{i=1}^{n} a\left(\omega_{i}\right) P\left(\omega_{i}\right),
$$

where $a_{i}=a\left(\omega_{i}\right)$ and $p_{i}=P\left(\omega_{i}\right)$ (with $P: \Omega \rightarrow \mathbb{R}$ being a probability distribution). Let us recall the standard way of translating the above formulation of the classical probability theory using the noncommutative framework of quantum theory. Note, that the space of complex random variables

$$
\psi: \Omega \longrightarrow \mathbb{C},
$$

defines a Hilbert space

$$
\mathcal{H}(\Omega):=\left\{\psi=\left(\psi_{1}, \ldots, \psi_{n}\right) \mid \psi_{i}=\psi\left(\omega_{i}\right)\right\} \cong \mathbb{C}^{n},
$$

equipped with the standard inner product

$$
\langle\varphi \mid \psi\rangle=\sum_{i=1}^{n} \bar{\varphi}_{i} \psi_{i}
$$

Now, any classical state $p \in S(\Omega)$ gives rise to a density matrix living in $\mathcal{H}(\Omega)$

$$
p=\left(p_{1}, \ldots, p_{n}\right) \longrightarrow \rho=\sum_{i=1}^{n} p_{i} e_{i i},
$$

where $e_{i i}=|i\rangle\langle i|$ and $e_{i} \equiv|i\rangle$ denotes an arbitrary (but fixed) orthonormal basis in $\mathcal{H}(\Omega)$. Hence classical states are represented by diagonal density matrices with respect to the same fixed basis $e_{i}$. Formula (2.7) defines therefore a decomposition of $\rho$ which coincides with the spectral decomposition when all the $p_{i}$ 's are different. Thus $S(\Omega)$ defines a commutative subalgebra of

$$
S(\Omega) \subset S\left(\mathbb{C}^{n}\right):=\left\{\rho: \mathbb{C}^{n} \longrightarrow \mathbb{C}^{n} \mid \rho \geq 0, \operatorname{Tr} \rho=1\right\},
$$

consisting of diagonal matrices. Classical pure states

$$
S_{0}(\Omega) \subset S_{0}\left(\mathbb{C}^{n}\right):=\left\{\rho \in S\left(\mathbb{C}^{n}\right) \mid \rho^{2}=\rho\right\},
$$

are represented by the rank-1 projectors $e_{i i}$. In the same way classical random variables define a commutative subalgebra of $\mathcal{B}\left(\mathbb{C}^{n}\right)$ :

$$
a=\left(a_{1}, \ldots, a_{n}\right) \longrightarrow a=\sum_{i=1}^{n} a_{i} e_{i i},
$$

that is, one may introduce the "classical" algebra $\mathcal{B}(\Omega) \subset \mathcal{B}\left(\mathbb{C}^{n}\right)$

$$
\mathcal{B}(\Omega):=\left\{a \in \mathcal{B}\left(\mathbb{C}^{n}\right) \mid a=\sum_{i=1}^{n} a_{i} e_{i i}\right\} .
$$

An element $a \in \mathcal{B}(\Omega)$ defines a classical observable iff $a^{*}=a$, i.e. $\overline{a_{i}}=a_{i}$. Finally, the classical formula (2.3) may be rewritten in a "quantum fashion" as follows

$$
\langle a, p\rangle=\operatorname{Tr} a \rho .
$$




\subsection{Classical channels}

A classical channel is a linear positive map

$$
\Lambda: \mathcal{B}\left(\Omega_{1}\right) \longrightarrow \mathcal{B}\left(\Omega_{2}\right)
$$

where $\Omega_{1}=\left\{\omega_{1}, \ldots, \omega_{n_{1}}\right\}$ and $\Omega=\left\{\varpi_{1}, \ldots, \varpi_{n_{2}}\right\}$. A channel is unital iff $\Lambda\left(\mathbb{I}_{1}\right)=\mathbb{I}_{2}$, where $\mathbb{I}_{k}$ denotes an identity element in the $\mathbb{C}^{*}$-algebra $\mathcal{B}\left(\Omega_{k}\right)$. Let

$$
\mathcal{B}\left(\Omega_{1}\right):=\left\{a \in \mathcal{B}\left(\mathbb{C}^{n_{1}}\right) \mid a=\sum_{i=1}^{n_{1}} a_{i} e_{i i}\right\}
$$

and

$$
\mathcal{B}\left(\Omega_{2}\right):=\left\{b \in \mathcal{B}\left(\mathbb{C}^{n_{2}}\right) \mid b=\sum_{i=1}^{n_{2}} b_{i} f_{i i}\right\},
$$

where $e_{i}$ defines an orthonormal basis in $\mathcal{H}\left(\Omega_{1}\right) \cong \mathbb{C}^{n_{1}}$, and $f_{i}$ defines an orthonormal basis in $\mathcal{H}\left(\Omega_{2}\right) \cong \mathbb{C}^{n_{2}}$. One obtains

$$
\Lambda(a)=\sum_{i=1}^{n_{1}} a_{i} \Lambda\left(e_{i i}\right)=\sum_{i=1}^{n_{1}} \sum_{j=1}^{n_{2}} a_{i} \Lambda_{i j} f_{j j}
$$

where

$$
\Lambda_{i j}:=\operatorname{Tr}\left(f_{j j} \Lambda\left(e_{i i}\right)\right) .
$$

A linear map $\Lambda$ is positive if and only if $\Lambda_{i j} \geq 0$. A channel $\Lambda$ transforms $a=\sum_{i} a_{i} e_{i i}$ into $b=\sum_{j} b_{j} f_{j j}$, with $b_{j}=\sum_{i} a_{i} \Lambda_{i j}$. It is represented by the $n_{1} \times n_{2}$ matrix $\left(\Lambda_{i j}\right)$ and it has the following Kraus representation

$$
\Lambda(a)=\sum_{i=1}^{n_{1}} \sum_{j=1}^{n_{2}} K_{i j} a K_{i j}^{*},
$$

where the operators $K_{i j}: \mathbb{C}^{n_{1}} \longrightarrow \mathbb{C}^{n_{2}}$ are defined by

$$
K_{i j}:=\sqrt{\Lambda_{i j}}\left|f_{j}\right\rangle\left\langle e_{i}\right|,
$$

and $\sqrt{\Lambda_{i j}}$ is any complex valued square root of $\left(\Lambda_{i j}\right)$. Let us observe that the above representation of the classical channel involves operators which are not diagonal. A channel is unital if

$$
\sum_{i=1}^{n_{1}} \Lambda_{i j}=1, \quad j=1, \ldots, n_{2} .
$$

It is therefore clear that a unital channel defines the conditional probability

$$
p_{i \mid j}:=\Lambda_{i j}
$$

on the space $\Omega_{1} \times \Omega_{2}$. 
Example 1 (Unitary channel) Consider a channel

$$
\Lambda: \mathcal{B}(\Omega) \longrightarrow \mathcal{B}(\Omega)
$$

defined by

$$
\Lambda(a):=U a U^{*},
$$

where $U$ is an $n \times n$ unitary matrix corresponding to a permutation $\pi$ from the symmetric group $S_{n}$, i.e.

$$
U_{i j}:=\delta_{i \pi(j)}, \quad i, j=1, \ldots, n
$$

One easily finds

$$
\Lambda(a)=\sum_{i=1}^{n} a_{i} e_{\pi(i) \pi(i)},
$$

where $\Lambda$ is represented by the following doubly stochastic matrix

$$
\Lambda_{i j}=\operatorname{Tr}\left(e_{j j} \Lambda\left(e_{i i}\right)\right)=\delta_{j \pi(i)} .
$$

Interestingly one has $\Lambda=U^{T}$.

Example 2 (Completely depolarizing channel) Consider the unital channel

$$
\Lambda: \mathcal{B}\left(\Omega_{1}\right) \longrightarrow \mathcal{B}\left(\Omega_{2}\right)
$$

defined by

$$
\Lambda(a)=\frac{1}{n_{1}} \mathbb{I}_{2} \operatorname{Tr} a
$$

corresponding to the matrix $\left(\Lambda_{i j}\right)$ given by:

$$
\Lambda_{i j}=\frac{1}{n_{1}}
$$

Note, that for $n_{1}=n_{2}$, the matrix $\left(\Lambda_{i j}\right)$ is doubly stochastic. The corresponding representation (2.18) reads as follows

$$
\Lambda(a)=\sum_{i=1}^{n_{1}} \sum_{j=1}^{n_{2}} K_{i j} a K_{i j}^{*},
$$

where the $K_{i j}$ are defined by (2.19).

\subsection{Composite classical systems}

Consider now a composite system consisting of two subsystems with state spaces $\Omega_{1}$ and $\Omega_{2}$. The corresponding space of elementary events has the form

$$
\Omega_{12}=\Omega_{1} \times \Omega_{2}
$$

hence $\left|\Omega_{12}\right|=n_{1} n_{2}$. The corresponding space of states consists of the joint probability distributions $P: \Omega_{12} \longrightarrow \mathbb{R}$

$$
S\left(\Omega_{12}\right):=\left\{p_{i j} \mid p_{i j} \geq 0, \sum_{i=1}^{n_{1}} \sum_{j=1}^{n_{2}} p_{i j}=1\right\}
$$


where $p_{i j}=P\left(\omega_{i}, \varpi_{j}\right)$. Again, one may translate multipartite case into the "quantum" framework by considering the complex random variables $\psi: \Omega_{12} \longrightarrow \mathbb{C}$ which give rise to the following Hilbert space

$$
\mathcal{H}\left(\Omega_{12}\right)=\mathcal{H}\left(\Omega_{1}\right) \otimes \mathcal{H}\left(\Omega_{2}\right) \cong \mathbb{C}^{n_{1}} \otimes \mathbb{C}^{n_{2}},
$$

hence the classical space $S\left(\Omega_{12}\right)$ defines a subspace of $S\left(\mathcal{H}\left(\Omega_{12}\right)\right)$

$$
S\left(\Omega_{12}\right) \subset S\left(\mathcal{H}\left(\Omega_{12}\right)\right):=\left\{\rho: \mathbb{C}^{n_{1}} \otimes \mathbb{C}^{n_{2}} \longrightarrow \mathbb{C}^{n_{1}} \otimes \mathbb{C}^{n_{2}} \mid \rho \geq 0, \operatorname{Tr} \rho=1\right\},
$$

consisting of diagonal matrices

$$
\rho=\sum_{i=1}^{n_{1}} \sum_{j=1}^{n_{2}} p_{i j} e_{i i} \otimes f_{j j} .
$$

Remark 1 (A classical analogue of the maximally entangled state) Consider a bipartite system with $\Omega_{12}=\Omega \times \Omega$ and $|\Omega|=n$. For any $U \in U(n)$ one defines a maximally entangled state in $\mathbb{C}^{n} \otimes \mathbb{C}^{n}$

$$
P_{U}:=\frac{1}{n} \sum_{i, j=1}^{n} e_{i j} \otimes U e_{i j} U^{*} .
$$

In the classical case the unitaries correspond to permutations from the symmetric group $S_{n}$ and one only considers diagonal states. Hence, the classical counterpart of $P_{U}$ is given by

$$
P_{\pi}=\frac{1}{n} \sum_{i=1}^{n} e_{i i} \otimes e_{\pi(i) \pi(i)} .
$$

Note, that contrarily to $P_{U}$, its classical counterpart $P_{\pi}$ is no longer a pure state. One may call $P_{\pi}$ a maximally correlated classical state.

Remark 2 (A classical analog of the Choi-Jamiołkowski isomorphism) There is an evident isomorphism between classical channels and classical states from $S(\Omega \times \Omega)$ : if $\Lambda: \mathcal{B}(\Omega) \longrightarrow$ $\mathcal{B}(\Omega)$ is a unital channel, then

$$
\rho=\frac{1}{n} \sum_{i=1}^{n} e_{i i} \otimes \Lambda^{\#}\left(e_{i i}\right),
$$

where $\Lambda^{\#}: \mathcal{S}(\Omega) \longrightarrow \mathcal{S}(\Omega)$ is the dual map, defines a classical state of the composite system in $\Omega \times \Omega$. Note, that

$$
\Lambda^{\#}\left(e_{i i}\right)=\sum_{j=1}^{n} p_{j \mid i} e_{j j}
$$

implies

$$
\rho=\sum_{i, j=1}^{n} p_{i j} e_{i i} \otimes e_{j j},
$$

where $p_{i j}$ is the joint probability defined by $p_{i j}=p_{i \mid j} p_{j}^{(0)}$, and $p^{(0)}$ corresponds to the uniform probability distribution $p_{k}^{(0)}=\frac{1}{n}$. 
Remark 3 (A classical analog of the teleportation protocol) The state $P_{\pi}$ may be used to perform a classical teleportation protocol: suppose that Alice would like to teleport a classical state

$$
\rho_{A}=\sum_{i=1}^{n} p_{i} e_{i i}
$$

In analogy to the quantum case one defines the joint state $\rho_{A} \otimes P_{\pi}$ living in $\mathcal{H}(\Omega) \otimes \mathcal{H}(\Omega) \otimes \mathcal{H}(\Omega)$ and performs the joint measurement of $n^{2} P_{0} \otimes \mathbb{I}_{B}$, where $P_{0}$ corresponds to the trivial permutation, i.e. $P_{0}=n^{-1} \sum_{i} e_{i i} \otimes e_{i i}$, and $\mathbb{I}_{B}$ is an identity operator in the Bob space. One obtains

$$
\rho_{B}=n^{2} \operatorname{Tr}_{12}\left(P_{0} \otimes \mathbb{I}_{B} \cdot \rho_{A} \otimes P_{\pi}\right)=\sum_{i=1}^{n} p_{\pi^{-1}(i)} e_{i i} .
$$

It is therefore clear that performing the unitary transformation corresponding to $\pi$ Bob recovers $\rho_{A}$. Summarizing: a classical teleportation channel consists in simple permutation $p_{i} \longrightarrow p_{\pi(i)}$, where the permutation $\pi$ corresponds to the 'maximally entangled state' $P_{\pi}$ used in the teleportation protocol. It is clear that there is a fundamental difference between classical and quantum teleportation: in the quantum case Bob needs 1 bit of additional classical information from Alice. It is no longer needed in the classical case. Knowing $\pi$ Bob reconstructs $\rho_{A}$ without any additional information.

Consider a classical system living in $\Omega$ and let $\rho$ be a classical state from $S(\Omega)$. To define a channel let $\sigma$ be a fixed state of the ancilla - again from $\Omega$ - and define

$$
\Lambda^{\#}(\rho)=\operatorname{Tr}_{2}\left(U \rho \otimes \sigma U^{*}\right),
$$

where $U$ is an $n^{2} \times n^{2}$ unitary matrix corresponding to a permutation from the symmetric group $S_{n^{2}}$. The simplest example corresponds to $n=2$ : let $\rho=\sum_{i} p_{i} e_{i i}$ and $\sigma=\sum_{i} q_{i} e_{i i}$. One finds the following $2^{2}=4$ channels

$$
p_{i} \longrightarrow \sum_{j=1}^{2} \Lambda_{i j}^{(k)} p_{j}
$$

with

$$
\Lambda^{(1)}=\left(\begin{array}{cc}
1 & 0 \\
0 & 1
\end{array}\right), \quad \Lambda^{(2)}=\left(\begin{array}{cc}
0 & 1 \\
1 & 0
\end{array}\right)
$$

and

$$
\Lambda^{(3)}=\left(\begin{array}{ll}
q_{1} & q_{2} \\
q_{2} & q_{1}
\end{array}\right)=q_{1} \Lambda^{(1)}+q_{2} \Lambda^{(2)}, \quad \Lambda^{(4)}=\left(\begin{array}{ll}
q_{2} & q_{1} \\
q_{1} & q_{2}
\end{array}\right)=q_{1} \Lambda^{(2)}+q_{2} \Lambda^{(1)}
$$

Note that all 4 matrices $\left[\Lambda_{i j}^{(k)}\right]$ are doubly stochastic. One easily proves the following general statement.

Proposition 1 A classical channel defined via the reduction procedure 2.43) is doubly stochastic.

We stress that a quantum channel defined by the reduction procedure is trace preserving but not necessarily unital. 


\subsection{Multipartite case}

The bipartite case may be easily generalized to many parties. Consider a composite system consisting of $N$ parties with

$$
\Omega_{N \ldots 1}=\Omega_{N} \times \ldots \times \Omega_{1}
$$

and $\left|\Omega_{k}\right|=n_{k}$. It implies $\left|\Omega_{1 \ldots N}\right|=n_{1} \ldots n_{N}$. The corresponding space of states consists of the joint probability distributions $P: \Omega_{N \ldots 1} \longrightarrow \mathbb{R}$

$$
S\left(\Omega_{N \ldots 1}\right):=\left\{p_{i_{N} \ldots i_{1}} \mid p_{i_{N} \ldots i_{1}} \geq 0, \quad \sum_{i_{1} \ldots i_{N}} p_{i_{N} \ldots i_{1}}=1\right\}
$$

where $p_{i_{N} \ldots i_{1}}=P\left(\omega_{i_{N}}^{(N)}, \ldots, \omega_{i_{1}}^{(1)}\right)$, and $\omega_{i}^{(k)}$ is an elementary event from $\Omega_{k}$.

Again, one may translate multipartite case into the quantum framework. Note that complex random variables

$$
\psi: \Omega_{N \ldots 1} \longrightarrow \mathbb{C},
$$

give rise to the multipartite Hilbert space

$$
\mathcal{H}\left(\Omega_{N \ldots 1}\right)=\mathcal{H}\left(\Omega_{N}\right) \otimes \ldots \otimes \mathcal{H}\left(\Omega_{1}\right) \cong \mathbb{C}^{n_{N}} \otimes \ldots \otimes \mathbb{C}^{n_{1}}
$$

and hence the classical space $S\left(\Omega_{N \ldots 1}\right)$ defines a subspace of $S\left(\mathcal{H}\left(\Omega_{N \ldots 1}\right)\right)$ consisting of the diagonal density matrices

$$
\rho=\sum_{i_{1}=1}^{n_{1}} \ldots \sum_{i_{N}=1}^{n_{N}} p_{i_{N} \ldots i_{1}} e_{i_{N} i_{N}}^{(N)} \otimes \ldots \otimes e_{i_{1} i_{1}}^{(1)}
$$

where $\left\{e_{1}^{(k)}, \ldots, e_{n_{k}}^{(k)}\right\}$ defines an orthonormal basis in $\mathcal{H}\left(\Omega_{k}\right)$.

\subsection{Markovian states}

Consider now the special case when all subsystems live on the same space $\Omega$. A classical $N$-partite state on $\Omega \times \ldots \times \Omega$

$$
\rho=\sum_{i_{N}, \ldots, i_{1}=1}^{n} p_{i_{N} \ldots i_{1}} e_{i_{N} i_{N}} \otimes \ldots \otimes e_{i_{1} i_{1}}
$$

is Markovian, if the $N$-partite joint probabilities $p_{i_{N} \ldots i_{1}}$ having the following property

$$
p_{i_{N} \ldots i_{1}}=p_{i_{N} \mid i_{N-1}} p_{i_{N-1} \mid i_{N-2}} \cdots p_{i_{2} \mid i_{1}} p_{i_{1}}
$$

where $p_{i \mid j}$ defines the conditional probability on $\Omega \times \Omega$ and $p_{i}$ is a probability distribution on $\Omega$. If $\rho$ is Markovian, then its reduction with respect to the subsystems $N, N-1, \ldots, N-k+1$ gives $(N-k)$-partite Markovian state

$$
\operatorname{Tr}_{N} \ldots \operatorname{Tr}_{N-k+1} \rho=\sum_{i_{N-k}, \ldots, i_{1}=1}^{n} p_{i_{N-k} \mid i_{N-k-1}} \ldots p_{i_{2} \mid i_{1}} p_{i_{1}} e_{i_{N-k} i_{N-k}} \otimes \ldots \otimes e_{i_{1} i_{1}} .
$$

Let us translate the Markovian property into the quantum noncommutative setting. Let $\mathcal{A}_{1}$ and $\mathcal{A}_{2}$ be $\mathbb{C}^{*}$-algebras. 
Definition 1 A linear completely positive map

$$
\mathcal{E}: \mathcal{A}_{2} \otimes \mathcal{A}_{1} \longrightarrow \mathcal{A}_{1}
$$

is called a transition expectation if

$$
\mathcal{E}\left(\mathbb{1}_{2} \otimes \mathbb{1}_{1}\right)=\mathbb{1}_{1}
$$

where $\mathbb{1}_{k}$ denotes an identity element in $\mathcal{A}_{k}$.

One has the following

Proposition 2 An $N$-partite state $\rho$ on $\Omega^{N}$ is Markovian if there exist a transition expectation

$$
\mathcal{E}: \mathcal{B}(\Omega) \otimes \mathcal{B}(\Omega) \longrightarrow \mathcal{B}(\Omega)
$$

such that

$$
\operatorname{Tr}\left(\rho\left(a_{N} \otimes \ldots \otimes a_{1}\right)\right)=\operatorname{Tr}\left(\rho_{1} \mathcal{E}\left(a_{N} \otimes \mathcal{E}\left(a_{N-1} \otimes \ldots \otimes \mathcal{E}\left(a_{1} \otimes \mathbb{I}\right)\right) \ldots\right)\right)
$$

is satisfied for arbitrary $a_{1}, \ldots, a_{N} \in \mathcal{B}(\Omega)$, where $\rho_{1}=\operatorname{Tr}_{1} \rho$ is the reduced density matrix living on the first subsystem.

Indeed, introducing so called Markov operator $P: \mathcal{B}(\Omega) \longrightarrow \mathcal{B}(\Omega)$

$$
P\left(e_{i i}\right)=\sum_{k=1}^{n} p_{i \mid j} e_{j j},
$$

where $p_{i \mid j}$ denotes conditional probability, and defining the transition expectation via

$$
\mathcal{E}\left(a_{2} \otimes a_{1}\right)=P\left(a_{2}\right) a_{1}
$$

one shows equivalence of (2.58) and (2.53).

\section{Classical liftings}

Let $\mathcal{A}_{1}$ and $\mathcal{A}_{2}$ be $\mathbb{C}^{*}$-algebras. One introduces [3] the following

Definition 2 A lifting from $\mathcal{A}_{1}$ to $\mathcal{A}_{2} \otimes \mathcal{A}_{1}$ is a map

$$
\mathcal{E}^{\#}: \mathcal{S}\left(\mathcal{A}_{1}\right) \longrightarrow \mathcal{S}\left(\mathcal{A}_{2} \otimes \mathcal{A}_{1}\right)
$$

If the map $\mathcal{E}^{\#}$ is affine and its dual $\mathcal{E}: \mathcal{A}_{2} \otimes \mathcal{A}_{1} \longrightarrow \mathcal{A}_{1}$ is completely positive, then one calls $\mathcal{E}^{\#}$ a linear lifting. If $\mathcal{E}^{\#}$ maps pure states into pure states, one calls it a pure lifting.

Following [3] let us consider a lifting

$$
\mathcal{E}^{\#}: \mathcal{S}\left(\Omega_{1}\right) \longrightarrow \mathcal{S}\left(\Omega_{2} \times \Omega_{1}\right)
$$


Note, that any linear lifting has the following form

$$
\mathcal{E}^{\#}(\rho)=\sum_{i, j=1}^{n_{1}} \sum_{k=1}^{n_{2}} p_{i} \mathcal{E}_{i j k} f_{j j} \otimes e_{k k},
$$

where

$$
\mathcal{E}_{i j k}:=\operatorname{Tr}\left(f_{j j} \otimes e_{k k} \mathcal{E}^{\#}\left(e_{i i}\right)\right)=\operatorname{Tr}\left(e_{i i} \mathcal{E}\left(f_{j j} \otimes e_{k k}\right)\right),
$$

and $\rho=\sum_{i} p_{i} e_{i i}$. One has

$$
\mathcal{E}^{\#}(\rho)=\sum_{j=1}^{n_{2}} \sum_{k=1}^{n_{1}} p_{j k} f_{j j} \otimes e_{k k}
$$

and hence

$$
p_{j k}=\sum_{i=1}^{n_{1}} \mathcal{E}_{i j k} p_{i}
$$

Definition 3 A lifting $\mathcal{E}^{\#}$ is called non-demolishing for a state $\rho \in \mathcal{S}\left(\Omega_{1}\right)$ if

$$
\operatorname{Tr}_{2} \mathcal{E}^{\#}(\rho)=\rho \text {. }
$$

It is clear that if $\mathcal{E}^{\#}$ is linear and non-demolishing for some $\rho$, then it is non-demolishing for all states. In this case one has

$$
\sum_{k=1}^{n_{2}} \mathcal{E}_{i j k}=\delta_{i j}
$$

Remark 4 The notion of nondemolition lifting defined here is essentially (i.e., up to minor technicalities) included in the more abstract notion of state extension introduced by Cecchini and Petz [28] (see also [29]).

Definition 4 A lifting $\mathcal{E}^{\#}$ is Markovian lifting if

$$
\mathcal{E}^{\#}\left(e_{i i}\right)=\sum_{j=1}^{n_{2}} p_{j \mid i} f_{j j} \otimes e_{i i}
$$

where $p_{j \mid i}$ stands for the conditional probability on $\Omega_{2} \times \Omega_{1}$.

Example 3 (Pure lifting) Let $s:\left\{1, \ldots, n_{1}\right\} \longrightarrow\left\{1, \ldots, n_{2}\right\}$ be a function and define

$$
\mathcal{E}^{\#}\left(e_{i i}\right)=f_{s(i) s(i)} \otimes e_{i i},
$$

that is, it lifts a pure state on $\Omega_{1}$ into pure states on $\Omega_{2} \times \Omega_{1}$. Hence, for $\rho=\sum_{i} p_{i} e_{i i}$ one finds

$$
\mathcal{E}^{\#}(\rho)=\sum_{i=1}^{n_{1}} p_{i} f_{s(i) s(i)} \otimes e_{i i},
$$

which implies

$$
\mathcal{E}_{i j k}=\delta_{i k} \delta_{j, s(i)}
$$


Example 1 (Product lifting) Let

$$
\mathcal{E}_{i j k}=\delta_{i k} q_{j}
$$

where $\sigma=\sum_{k} q_{k} f_{k k}$ is a classical state over $\Omega_{2}$. One obtains

$$
\mathcal{E}^{\#}(\rho)=\sigma \otimes \rho
$$

that is, a joint probability is given by a product formula $p_{j k}=p_{j} q_{k}$.

Example 2 Let $\sigma$ be a fixed state on $\Omega_{2}$ and

$$
\Gamma: \mathcal{B}\left(\Omega_{2} \times \Omega_{1}\right) \longrightarrow \mathcal{B}\left(\Omega_{2} \times \Omega_{1}\right),
$$

be a linear positive map. Define the lifting $\mathcal{E}^{\# \Gamma, \sigma}$ by

$$
\mathcal{E}^{\# \Gamma, \sigma}(\rho):=\Gamma^{\#}(\sigma \otimes \rho) .
$$

Note, that if $\Gamma=\mathrm{id}_{2} \otimes \mathrm{id}_{1}$, then the above formula recovers a product lifting.

Example 3 (Classical Ohya lifting) If

$$
\mathcal{E}_{i j k}=\delta_{i k} \delta_{j k}
$$

then

$$
\mathcal{E}^{\#}(\rho)=\sum_{i=1}^{n} p_{i} e_{i i} \otimes e_{i i} .
$$

Note, that a characteristic feature of the Ohya lifting is that both reduced states reproduce the original state $\rho$, i.e.

$$
\operatorname{Tr}_{1} \mathcal{E}^{\#}(\rho)=\operatorname{Tr}_{2} \mathcal{E}^{\#}(\rho)=\rho,
$$

for an arbitrary classical state $\rho$ on $\Omega$. It shows that Ohya lifting realizes a perfect classical cloning machine [31]. Moreover, since both reduced states are the same Ohya lifting provides at the same time a protocol for a classical broadcasting [32].

\section{Multipartite classical liftings}

It is clear that a lifting (3.1) may be easily generalized to the multipartite case: consider a set $\mathcal{A}_{1}, \ldots, \mathcal{A}_{N}$ of $\mathbb{C}^{*}$-algebras.

Definition 5 An $N$-lifting from $\mathcal{A}_{1}$ to $\mathcal{A}_{N} \otimes \ldots \otimes \mathcal{A}_{1}$ is a map

$$
\mathcal{E}^{\#}: \mathcal{S}\left(\mathcal{A}_{1}\right) \longrightarrow \mathcal{S}\left(\mathcal{A}_{N} \otimes \ldots \otimes \mathcal{A}_{1}\right) \text {. }
$$

If the map $\mathcal{E}^{\#}$ is affine and its dual $\mathcal{E}: \mathcal{A}_{N} \otimes \ldots \otimes \mathcal{A}_{1} \longrightarrow \mathcal{A}_{1}$ is completely positive, then one calls $\mathcal{E}^{\#}$ a linear lifting. 
Applying the above definition to the classical commutative case one finds

$$
\mathcal{E}_{N}^{\#}: \mathcal{S}\left(\Omega_{1}\right) \longrightarrow \mathcal{S}\left(\Omega_{N} \times \ldots \times \Omega_{1}\right)
$$

Note, that any linear classical $N$-lifting has the following form

$$
\mathcal{E}_{N}^{\#}(\rho)=\sum_{i=1}^{n_{1}} \sum_{i_{1}=1}^{n_{1}} \ldots \sum_{i_{N}=1}^{n_{N}} \mathcal{E}_{i_{N} \ldots i_{1} i} p_{i} e_{i_{N} i_{N}}^{(N)} \otimes \ldots \otimes e_{i_{1} i_{1}}^{(1)},
$$

where

$$
\mathcal{E}_{i_{N} \ldots i_{1} i}:=\operatorname{Tr}\left(e_{i_{N} i_{N}}^{(N)} \otimes \ldots \otimes e_{i_{1} i_{1}}^{(1)} \mathcal{E}_{N}^{\#}\left(e_{i i}^{(1)}\right)\right)=\operatorname{Tr}\left(\mathcal{E}_{N}\left(e_{i_{N} i_{N}}^{(N)} \otimes \ldots \otimes e_{i_{1} i_{1}}^{(1)}\right) e_{i i}^{(1)}\right)
$$

and $\rho=\sum_{i} p_{i} e_{i i}$. Note, that $\mathcal{E}_{N}^{\#}(\rho)$ is an $N$-partite classical state and hence it corresponds to an $N$-partite joint probability distribution $p_{i_{n} \ldots i_{1}}$, that is

$$
\mathcal{E}_{N}^{\#}(\rho)=\sum_{i_{1}=1}^{n_{1}} \ldots \sum_{i_{N}=1}^{n_{N}} p_{i_{N} \ldots i_{1}} e_{i_{N} i_{N}}^{(N)} \otimes \ldots \otimes e_{i_{1} i_{1}}^{(1)},
$$

and hence

$$
p_{i_{N} \ldots i_{1}}=\sum_{i_{1}=1}^{n_{1}} \mathcal{E}_{i_{N} \ldots i_{1} i} p_{i}
$$

Example 4 (Pure $N$-lifting) Let $s_{k}:\left\{1, \ldots, n_{1}\right\} \longrightarrow\left\{1, \ldots, n_{k}\right\}(k=2,3, \ldots, N)$ be a family of functions and define the following linear $N$-lifting

$$
\mathcal{E}_{N}^{\#}\left(e_{i i}^{(1)}\right)=e_{s_{N}(i) s_{N}(i)}^{(N)} \otimes \ldots \otimes e_{s_{2}(i) s_{2}(i)}^{(2)} \otimes e_{i i}^{(1)}
$$

that is, it lifts a pure state on $\Omega_{1}$ into pure states on $\Omega_{N} \times \ldots \times \Omega_{1}$. Hence, for $\rho=\sum_{i} p_{i} e_{i i}^{(1)}$ one finds

$$
\mathcal{E}_{N}^{\#}(\rho)=\sum_{i=1}^{n_{1}} p_{i} e_{s_{N}(i) s_{N}(i)}^{(N)} \otimes \ldots \otimes e_{s_{2}(i) s_{2}(i)}^{(2)} \otimes e_{i i}^{(1)}
$$

which implies

$$
\mathcal{E}_{i_{N} \ldots i_{1} i}=\delta_{i_{N}, s_{N}(i)} \ldots \delta_{i_{2}, s_{2}(i)} \delta_{i_{1} i}
$$

Example 4 (Product $N$-lifting) Let $\sigma_{N} \otimes \ldots \otimes \sigma_{2}$ be a product state on $\Omega_{N} \times \ldots \times \Omega_{2}$. One defines

$$
\mathcal{E}_{N}^{\#}(\rho)=\sigma_{N} \otimes \ldots \otimes \sigma_{2} \otimes \rho,
$$

that is,

$$
\mathcal{E}_{i_{N} \ldots i_{1} i}=q_{i_{N}}^{(N)} \ldots q_{i_{2}}^{(2)} \delta_{i_{1} i}
$$

where $\sigma_{k}=\sum_{i_{k}} q_{i_{k}}^{(k)} e_{i_{k} i_{k}}^{(k)}$. It is clear that the corresponding joint probability factorizes as follows

$$
p_{i_{N} \ldots i_{1}}=q_{i_{N}}^{(N)} \ldots q_{i_{2}}^{(2)} p_{i_{1}} .
$$


Example 5 Let $\sigma=\sigma_{N} \otimes \ldots \otimes \sigma_{2}$ be a product state on $\Omega_{N} \times \ldots \times \Omega_{2}$ and

$$
\Gamma: \mathcal{B}\left(\Omega_{N} \times \ldots \times \Omega_{1}\right) \longrightarrow \mathcal{B}\left(\Omega_{N} \times \ldots \times \Omega_{1}\right),
$$

be a linear positive map. Define the $N$-lifting $\mathcal{E}^{\# \Gamma, \sigma}$ by

$$
\mathcal{E}_{N}^{\# \Gamma, \sigma}(\rho):=\Gamma^{\#}(\sigma \otimes \rho) .
$$

Note, that if $\Gamma=\mathrm{id}_{N} \otimes \ldots \otimes \mathrm{id}_{1}$, then the above formula recovers a product $N$-lifting.

Example 6 (Classical Ohya $N$-lifting) Let $\Omega_{1}=\ldots=\Omega_{N}=\Omega$. Taking

$$
\mathcal{E}_{i_{N} \ldots i_{1} i}=\delta_{i_{N} i} \ldots \delta_{i_{1} i}
$$

one obtains

$$
\mathcal{E}_{N}^{\#}\left(e_{i i}\right)=e_{i i} \otimes \ldots \otimes e_{i i},
$$

and hence for $\rho=\sum_{i} p_{i} e_{i i}$ one finds

$$
\mathcal{E}_{N}^{\#}(\rho)=\sum_{i=1}^{n} p_{i} e_{i i} \otimes \ldots \otimes e_{i i} .
$$

Note, that a characteristic feature of the Ohya lifting is that all reduced one-partite states reproduce the original state $\rho$, i.e.

$$
\operatorname{Tr}_{\breve{1}} \mathcal{E}^{\#}(\rho)=\ldots=\operatorname{Tr}_{\breve{N}} \mathcal{E}^{\#}(\rho)=\rho,
$$

where $\operatorname{Tr}_{\breve{k}}$ denotes the partial trace with respect all subsystems excluding $k$ th. It shows that Ohya lifting realizes a perfect classical cloning machine 31. Moreover, since both reduced states are the same Ohya lifting provides at the same time a protocol for a classical broadcasting [32].

Remark 1 Let $\varphi_{k}: \mathcal{B}\left(\mathbb{C}^{n}\right) \longrightarrow \mathcal{B}\left(\mathbb{C}^{n}\right)$ be a set of positive unital maps (for $\left.k=1, \ldots, N\right)$. Then the following $N$-partite state

$$
\rho=\sum_{i=1}^{n} p_{i} \varphi_{1}^{\#}\left(e_{i i}\right) \otimes \ldots \otimes \varphi_{N}^{\#}\left(e_{i i}\right),
$$

is a (quantum) $N$-separable state.

Let us observe that in the special case when $\Omega_{2}=\ldots=\Omega_{N}=: \Omega$ there is a simple way to construct an $N$-lifting out of the lifting

$$
\mathcal{E}^{\#}: \mathcal{S}\left(\Omega_{1}\right) \longrightarrow \mathcal{S}\left(\Omega_{2} \times \Omega_{1}\right),
$$

Define the $N$-lifting

$$
\mathcal{E}_{N}^{\#}: \mathcal{S}\left(\Omega_{1}\right) \longrightarrow \mathcal{S}\left(\Omega_{N} \times \ldots \times \Omega_{1}\right)
$$

by the following recurrence formula: for $N=3$

$$
\mathcal{E}_{3}^{\#}: \mathcal{S}\left(\Omega_{1}\right) \longrightarrow \mathcal{S}\left(\Omega_{3} \times \Omega_{2} \times \Omega_{1}\right),
$$

one has

and for $N>3$

$$
\mathcal{E}_{3}^{\#}=\left(\mathrm{id}_{2} \otimes \mathcal{E}^{\#}\right) \circ \mathcal{E}^{\#},
$$

$$
\mathcal{E}_{N}^{\#}=\left(\mathrm{id}_{N-1} \otimes \ldots \otimes \mathrm{id}_{2} \otimes \mathcal{E}^{\#}\right) \circ \mathcal{E}_{N-1}^{\#} .
$$

An example of this construction is provided by the Ohya $N$-lifting. 


\section{Quantum noncommutative liftings}

\section{$5.1 \quad$ Linear liftings}

To any transition expectation represented by a completely positive linear unital map

$$
\mathcal{E}: \mathcal{A}_{2} \otimes \mathcal{A}_{1} \longrightarrow \mathcal{A}_{1}
$$

there corresponds a linear lifting

$$
\mathcal{E}^{\#}: S\left(\mathcal{A}_{1}\right) \longrightarrow S\left(\mathcal{A}_{2} \otimes \mathcal{A}_{1}\right) .
$$

Linear liftings play important role in the quantum theory of open systems. It is well known that a linear lifting which is nondemolishing for all states $\rho$ has the following form

$$
\mathcal{E}^{\#}(\rho)=\omega \otimes \rho,
$$

for some fixed state $\omega$ over $\mathcal{A}_{2}$. Let us assume that $\mathcal{A}_{k}=\mathcal{B}\left(\mathcal{H}_{k}\right)$. A product lifting (or assignment maps [9, 7]) defined by (5.3) gives rise to a linear completely positive map

$$
\Lambda^{\#}: S\left(\mathcal{A}_{1}\right) \longrightarrow S\left(\mathcal{A}_{1}\right)
$$

defined by

$$
\Lambda^{\#}(\rho):=\operatorname{Tr}_{2}\left[U(\omega \otimes \rho) U^{*}\right],
$$

where $U$ is a unitary operator in $\mathcal{H}_{2} \otimes \mathcal{H}_{1}$. It is well known that any linear completely positive map may be obtained this way.

Remark 5 Let us observe that linear lifting may be used to construct new classes of positive maps which are not completely positive. Let $\mathcal{E}^{\#}$ be a product lifting from $\mathcal{A}_{1}$ to $\mathcal{A}_{2} \otimes \mathcal{A}_{1}$ and let

$$
\psi: \mathcal{S}\left(\mathcal{A}_{2} \otimes \mathcal{A}_{1}\right) \longrightarrow \mathcal{S}\left(\mathcal{A}_{2} \otimes \mathcal{A}_{1}\right)
$$

be a positive map. Define a new map $\varphi: \mathcal{S}\left(\mathcal{A}_{1}\right) \longrightarrow \mathcal{S}\left(\mathcal{A}_{1}\right)$ via

$$
\varphi(\rho):=\operatorname{Tr}_{2}\left[\psi\left(\mathcal{E}^{\#}(\rho)\right)\right]=\operatorname{Tr}_{2}[\psi(\omega \otimes \rho)]
$$

where $\omega$ is a fixed state in $\mathcal{A}_{2}$. By construction $\varphi$ is a linear positive map. Note, however, that if $\psi$ is only positive but not completely positive then $\varphi$ needs not be completely positive. As an example consider $\mathcal{A}_{1}=\mathcal{A}_{2}=M_{2}(\mathbb{C})$ and let $\psi: M_{4}(\mathbb{C}) \longrightarrow M_{4}(\mathbb{C})$ be the Robertson map defined as follows [18, 21]

$$
\psi\left(\begin{array}{c|c}
X_{11} & X_{12} \\
\hline X_{21} & X_{22}
\end{array}\right)=\frac{1}{2}\left(\begin{array}{c|c}
\mathbb{I}_{2} \operatorname{Tr} X_{22} & X_{12}+R_{2}\left(X_{21}\right) \\
\hline X_{21}+R_{2}\left(X_{12}\right) & \mathbb{I}_{2} \operatorname{Tr} X_{11}
\end{array}\right)
$$

where we represented an element $X \in M_{4}(\mathbb{C})$ in the block form $X=\sum_{i, j=1}^{2} e_{i j} \otimes X_{i j}$ with $X_{i j} \in$ $M_{2}(\mathbb{C})$. Finally, $R_{2}$ denotes the reduction map $R_{2}: M_{2}(\mathbb{C}) \longrightarrow M_{2}(\mathbb{C})$ defined by

$$
R_{2}(X)=\mathbb{I}_{2} \operatorname{Tr} X-X,
$$


which is known to positive but not completely positive. Now, if $\mathcal{E}^{\#}$ is a product lifting $\mathcal{E}^{\#}(\rho)=$ $\omega \otimes \rho$, then one finds

$$
\varphi(\rho)=\frac{1}{2}\left(\begin{array}{cc}
2 \rho_{22} & \rho_{12}+\rho_{21} \\
\rho_{12}+\rho_{21} & 2 \rho_{11}
\end{array}\right) .
$$

Interestingly, the positive map $\varphi$ does not depend upon the state $\omega$. To show that $\varphi$ is not completely positive let us observe that

$$
(\mathrm{id} \otimes \varphi) P_{2}^{+}=\frac{1}{4}\left(\begin{array}{cc|cc}
0 & 0 & 0 & 1 \\
0 & 2 & 1 & 0 \\
\hline 0 & 1 & 2 & 0 \\
1 & 0 & 0 & 0
\end{array}\right),
$$

is not positively defined $\left(P_{2}^{+}\right.$denotes the maximally entangled state in $\mathbb{C}^{2} \otimes \mathbb{C}^{2}$, i.e. $P_{2}^{+}=$ $\left.\frac{1}{2} \sum_{i, j=1}^{2} e_{i j} \otimes e_{i j}\right)$.

\subsection{Nonlinear liftings}

Let $\Lambda$ be a linear completely positive unital map

$$
\Lambda: \mathcal{B}(\mathcal{H}) \longrightarrow \mathcal{B}(\mathcal{H}) \text {. }
$$

Definition 6 ([34]) A positive operator

$$
\pi:=\sum_{i, j=1}^{d} e_{i j} \otimes \Lambda\left(e_{i j}\right),
$$

living in $\mathcal{H}_{2} \otimes \mathcal{H}_{1}\left(\mathcal{H}_{k}=\mathbb{C}^{d}\right)$ is called the quantum conditional probability (QCP) operator.

QCP generalizes classical conditional probability for the quantum noncommutative case 11 Note, that in the classical case one may define

$$
\pi:=\sum_{i=1}^{d} e_{i i} \otimes \Lambda\left(e_{i i}\right),
$$

with

$$
\Lambda\left(e_{i i}\right)=\sum_{j=1}^{d} p_{i \mid j} e_{j j} .
$$

Then

$$
\sum_{i} p_{i \mid j}=1
$$

is equivalent to

$$
\operatorname{Tr}_{2} \pi=\mathbb{I},
$$

which follows from $\Lambda(\mathbb{I})=\mathbb{I}$. Following [34] let us define a nonlinear lifting by

$$
\mathcal{E}^{\#}(\rho):=\left(\mathbb{I} \otimes \rho^{\frac{1}{2}}\right) \pi\left(\mathbb{I} \otimes \rho^{\frac{1}{2}}\right) .
$$

\footnotetext{
${ }^{1}$ For slightly different approach to quantum conditional probability see $[35$ and 36 .
} 
Proposition 3 A lifting $\mathcal{E}^{\#}$ satisfies

$$
\operatorname{Tr}_{2} \mathcal{E}^{\#}(\rho)=\rho
$$

and

$$
\operatorname{Tr}_{1} \mathcal{E}^{\#}(\rho)=\Lambda^{\#}(\rho)^{\mathrm{T}}
$$

Remark 2 Note, that in the special case if

$$
\Lambda(x)=\mathbb{I} \operatorname{Tr} \omega^{T} x,
$$

for some density operator $\omega$ in $\mathcal{H}_{2}$, then (5.16) defines a linear product lifting

$$
\mathcal{E}^{\#}(\rho)=\omega \otimes \rho \text {. }
$$

Example 5 Let $\Lambda(a)=U a U^{*}$, with unitary $U$. One has for the lifting

$$
\begin{aligned}
\mathcal{E}^{\#}(\rho) & =\left(\mathbb{I} \otimes \rho^{\frac{1}{2}}\right) \sum_{i, j=1}^{n} e_{i j} \otimes U e_{i j} U^{*}\left(\mathbb{I} \otimes \rho^{\frac{1}{2}}\right) \\
& =\sum_{i, j=1}^{n} e_{i j} \otimes \rho^{\frac{1}{2}} U e_{i j} U^{*} \rho^{\frac{1}{2}}
\end{aligned}
$$

that is

$$
\mathcal{E}^{\#}(\rho)=\sum_{i, j=1}^{n} e_{i j} \otimes \phi\left(e_{i j}\right),
$$

where $\phi$ is the $\rho$-dependent CP map

$$
\phi(a)=K a K^{*},
$$

with the $\rho$-dependent operator given by $K=\rho^{\frac{1}{2}} U$.

Example 7 Let $\Lambda(a)=\sum_{i} \operatorname{Tr}\left(a e_{i i}\right) e_{i i}$. It leads to the following lifting

$$
\mathcal{E}^{\#}(\rho)=\sum_{i=1}^{n} e_{i i} \otimes \rho^{\frac{1}{2}} e_{i i} \rho^{\frac{1}{2}} .
$$

Note, that if $e_{i}$ defines eigen-basis for $\rho$, then

$$
\mathcal{E}^{\#}(\rho)=\sum_{i=1}^{n} \rho_{i} e_{i i} \otimes e_{i i},
$$

with $\rho_{i}$ being an eigenvalue of $\rho$, i.e. $\rho e_{i}=\rho_{i} e_{i}$. The above formula reproduces the nonlinear Ohya lifting. Recall, that the nonlinear Ohya lifting $\mathcal{E}^{\#}: \mathcal{S}(\mathcal{A}) \longrightarrow \mathcal{S}(\mathcal{A} \otimes \mathcal{A})$ is defined as follows [3]

$$
\mathcal{E}^{\#}(\rho)=\sum_{k} p_{k} E_{k} \otimes E_{k}
$$

where

$$
\rho=\sum_{k} p_{k} E_{k}
$$

stands for the spectral decomposition of $\rho$. We stress that $\mathcal{E}^{\#}$ is nonlinear since both $p_{k}$ and $E_{k}$ are $\rho$-dependent. 
Remark 3 Note, that a characteristic feature of the Ohya lifting is that both reduced states reproduce the original state $\rho$, i.e.

$$
\operatorname{Tr}_{1} \mathcal{E}^{\#}(\rho)=\operatorname{Tr}_{2} \mathcal{E}^{\#}(\rho)=\rho
$$

for an arbitrary classical state $\rho$ on $\Omega$. It shows that Ohya lifting realizes a perfect classical cloning machine [31]. Moreover, since both reduced states are the same Ohya lifting provides at the same time a protocol for a classical broadcasting [32].

Remark 4 Let $\theta$ be a compound state in $\mathcal{H}_{2} \otimes \mathcal{H}_{1}$ with marginals $\rho$ and $\sigma$. If $\phi$ is a CP map such that

$$
\theta=\sum_{i, j=1}^{n} e_{i j} \otimes \phi\left(e_{i j}\right)
$$

then one can rewrite $\theta$ as follows

$$
\theta=\sum_{i, j=1}^{n} e_{i j} \otimes \rho^{\frac{1}{2}} \Lambda\left(e_{i j}\right) \rho^{\frac{1}{2}},
$$

where $\Lambda$ is a unital CP map defined by

$$
\Lambda(a)=\rho^{-\frac{1}{2}} \phi(a) \rho^{-\frac{1}{2}}
$$

where we assume that $\rho$ is faithful state, i.e. $\rho>0$.

Suppose now that we have two QCP operators $\pi_{1}$ and $\pi_{2}$. Let us define a 3-partite operator $\pi_{1} \circ \pi_{2}$

$$
\pi_{1} \circ \pi_{2}: \mathcal{H}_{3} \otimes \mathcal{H}_{2} \otimes \mathcal{H}_{1} \longrightarrow \mathcal{H}_{3} \otimes \mathcal{H}_{2} \otimes \mathcal{H}_{1}
$$

with $\mathcal{H}_{k}=\mathbb{C}^{d}$, by

$$
\pi_{1} \circ \pi_{2}:=\left(\mathbb{I} \otimes \pi_{1}^{\frac{1}{2}}\right)\left(\pi_{2} \otimes \mathbb{I}\right)\left(\mathbb{I} \otimes \pi_{1}^{\frac{1}{2}}\right) .
$$

Proposition 4 The operator $\pi_{1} \circ \pi_{2}$ is positive and it satisfies the following basic properties:

$$
\begin{aligned}
\operatorname{Tr}_{3}\left(\pi_{1} \circ \pi_{2}\right) & =\pi_{1}, \\
\operatorname{Tr}_{32}\left(\pi_{1} \circ \pi_{2}\right) & =\mathbb{I} .
\end{aligned}
$$

It is clear that for $\pi_{1}=\pi_{2}=\pi$, the operator $\pi \circ \pi$ may be used to define a lifting from $M_{d}^{+}$ into $M_{d}^{+} \otimes M_{d}^{+} \otimes M_{d}^{+}$. One defines

$$
\mathcal{E}_{3 \mid 1}^{\#}(\rho)=\left(\mathbb{I} \otimes \mathbb{I} \otimes \rho^{\frac{1}{2}}\right)(\pi \circ \pi)\left(\mathbb{I} \otimes \mathbb{I} \otimes \rho^{\frac{1}{2}}\right) .
$$

Note, that due to (5.33) one has

$$
\operatorname{Tr}_{3} \mathcal{E}_{3 \mid 1}^{\#}(\rho)=\mathcal{E}_{2 \mid 1}^{\#}(\rho)
$$

where $\mathcal{E}_{2 \mid 1}^{\#}(\rho):=\mathcal{E}^{\#}(\rho)$ is defined in (5.16). This procedure may be immediately generalized for arbitrary $N$. One has the following recurrence formula for the $N$-partite operator

$$
\pi_{1} \circ \ldots \circ \pi_{N-1}: \mathcal{H}_{N} \otimes \ldots \otimes \mathcal{H}_{1} \longrightarrow \mathcal{H}_{N} \otimes \ldots \otimes \mathcal{H}_{1}
$$




$$
\pi_{1} \circ \ldots \circ \pi_{N-1}:=\left(\mathbb{I} \otimes \ldots \otimes \mathbb{I} \otimes \pi_{1}^{\frac{1}{2}}\right)\left(\pi_{2} \circ \ldots \circ \pi_{N-1} \otimes \mathbb{I}\right)\left(\mathbb{I} \otimes \ldots \otimes \mathbb{I} \otimes \pi_{1}^{\frac{1}{2}}\right) .
$$

One easily proves

$$
\begin{aligned}
\operatorname{Tr}_{N}\left(\pi_{1} \circ \ldots \circ \pi_{N-1}\right) & =\pi_{1} \circ \ldots \circ \pi_{N-2}, \\
\operatorname{Tr}_{N, N-1}\left(\pi_{1} \circ \ldots \circ \pi_{N-1}\right) & =\pi_{1} \circ \ldots \circ \pi_{N-3}, \\
& \vdots \\
\operatorname{Tr}_{N, \ldots, 2}\left(\pi_{1} \circ \ldots \circ \pi_{N-1}\right) & =\mathbb{I} .
\end{aligned}
$$

Again, when $\pi_{1}=\ldots=\pi_{N-1}=\pi$, then $N$-partite $\pi \circ \ldots \circ \pi$ is positive and one defines

$$
\mathcal{E}_{N \mid 1}^{\#}(\rho)=\left(\mathbb{I} \otimes \ldots \otimes \mathbb{I} \otimes \rho^{\frac{1}{2}}\right)(\pi \circ \ldots \circ \pi)\left(\mathbb{I} \otimes \ldots \otimes \mathbb{I} \otimes \rho^{\frac{1}{2}}\right) .
$$

It is clear that

$$
\operatorname{Tr}_{N} \mathcal{E}_{N \mid 1}^{\#}(\rho)=\mathcal{E}_{N-1 \mid 1}^{\#}(\rho)
$$

Note, that in the classical case defined one finds

$$
\mathcal{E}_{N \mid 1}^{\#}(\rho)=\sum_{i_{1}, \ldots, i_{N}=1}^{d} p_{i_{N} \mid i_{N-1}} \ldots p_{i_{2} \mid i_{1}} p_{i_{1}} e_{i_{N} i_{N}} \otimes \ldots \otimes e_{i_{1} i_{1}}
$$

where $\rho=\sum_{i} p_{i} e_{i i}$. Hence, the lifted $N$-partite state $\mathcal{E}^{\#}(\rho)$ generalizes classical Markovian state (for entanglement properties of a particular class of pure Markovian multipartite state see [30]).

\section{Circulant liftings}

In this section we analyze a particular class of liftings defined in terms of circulant states [23] (see also [24]). Circulant states play important role in Quantum Information Theory since majority of states of composite quantum systems considered in the literature turn out to be circulant states. The most important example of circulant states is provided by generalized Bell states. Let us mention also the class of isotropic states, Werner states, Bell diagonal states and many others (see 23] for more examples). Therefore, it is interesting to investigate the class of liftings directly related to the class of circulant states. This section provides also the illustration of the theoretical concepts introduced so far. It is organized as follows: we start with the notion of circulant decomposition of any Hilbert space being the tensor product $\mathbb{C}^{d} \otimes \mathbb{C}^{d}$. It turns out that it may be represented as a direct product of $d$ subspaces each of dimension $d$. This decomposition (we call it cyclic decomposition) provides starting point in the construction of a circulant state. Having defined circulant state we analyze the class of liftings such that $\mathcal{E}^{\#}(\rho)$ is a circulant state for any $\rho$ living in $\mathbb{C}^{d}$ and illustrate the construction by the special class of Bell diagonal liftings.

\subsection{Circulant decompositions}

The basic idea is to decompose the total Hilbert space $\mathbb{C}^{d} \otimes \mathbb{C}^{d}$ into a direct sum of $d$ orthogonal $d$-dimensional subspaces related by a certain cyclic property. Let us introduce a $d$-dimensional subspace

$$
\Sigma_{0}=\operatorname{span}\left\{e_{0} \otimes e_{0}, e_{1} \otimes e_{1}, \ldots, e_{d-1} \otimes e_{d-1}\right\}
$$


where $\left\{e_{0}, \ldots, e_{d-1}\right\}$ denote the standard computational basis in $\mathbb{C}^{d}$, together with the shift operator $S: \mathbb{C}^{d} \longrightarrow \mathbb{C}^{d}$ defined by

$$
S e_{k}=e_{k+1}, \quad \bmod d .
$$

Now, for any $\alpha=0, \ldots, d-1$, we define

$$
\Sigma_{\alpha}=\left(\mathbb{1} \otimes S^{\alpha}\right) \Sigma_{0}
$$

It is clear that $\Sigma_{\alpha}$ and $\Sigma_{\beta}$ are mutually orthogonal (for $\alpha \neq \beta$ ). Moreover,

$$
\Sigma_{0} \oplus \ldots \oplus \Sigma_{d-1}=\mathbb{C}^{d} \otimes \mathbb{C}^{d} .
$$

Due to the cyclic property of the shift operator (6.2), we call (6.4) a circulant decomposition [23]. For example in the case of two qubits $(d=2)$ one has

$$
\begin{aligned}
& \Sigma_{0}=\operatorname{span}\left\{e_{0} \otimes e_{0}, e_{1} \otimes e_{1}\right\}, \\
& \Sigma_{1}=\operatorname{span}\left\{e_{0} \otimes e_{1}, e_{1} \otimes e_{0}\right\},
\end{aligned}
$$

whereas for two qutrits $(d=3)$ one obtains

$$
\begin{aligned}
& \Sigma_{0}=\operatorname{span}\left\{e_{0} \otimes e_{0}, e_{1} \otimes e_{1}, e_{2} \otimes e_{2}\right\}, \\
& \Sigma_{1}=\operatorname{span}\left\{e_{0} \otimes e_{1}, e_{1} \otimes e_{2}, e_{2} \otimes e_{0}\right\}, \\
& \Sigma_{2}=\operatorname{span}\left\{e_{0} \otimes e_{2}, e_{1} \otimes e_{0}, e_{2} \otimes e_{1}\right\} .
\end{aligned}
$$

\subsection{Circulant states}

The circulant decomposition (6.4) gives rise to the following construction of a state in $\mathbb{C}^{d} \otimes \mathbb{C}^{d}$ : let $a^{(0)}, \ldots, a^{(d-1)}$ be a set of $d$ positive $d \times d$ matrices. Let us observe that

$$
\rho^{(\alpha)}=\sum_{i, j=0}^{d-1} a_{i j}^{(\alpha)} e_{i j} \otimes S^{\alpha} e_{i j} S^{* \alpha}, \quad \alpha=0, \ldots, d-1
$$

is a positive operator in $\mathbb{C}^{d} \otimes \mathbb{C}^{d}$ supported on $\Sigma_{\alpha}$. Hence, the following sum

$$
\rho=\rho^{(0)}+\ldots+\rho^{(d-1)},
$$

gives rise to a positive operator in $\mathbb{C}^{d} \otimes \mathbb{C}^{d}$. It defines a legitimate state iff $\operatorname{Tr} \rho=1$, which is equivalent to

$$
\operatorname{Tr}\left[a^{(0)}+\ldots+a^{(d-1)}\right]=1 .
$$

For obvious reason we call (6.8) a circulant state. This simple construction recovers many well known bipartite states from the literature (see [23]).

Consider now a partial transposition of the circulant state (6.8). It turns out that $\rho^{\Gamma}=(\mathbb{1} \otimes \tau) \rho$ is again circulant but it corresponds to another cyclic decomposition of the original Hilbert space $\mathbb{C}^{d} \otimes \mathbb{C}^{d}$. Recall, that $\rho$ is PPT (Positive Partial Transpose) if $\rho^{\Gamma} \geq 0$. Let us introduce the following permutation $\pi$ from the symmetric group $S_{d}$ :

$$
\pi(0)=0, \quad \pi(i)=d-i, \quad i=1,2, \ldots, d-1 .
$$


We use $\pi$ to introduce

$$
\widetilde{\Sigma}_{0}=\operatorname{span}\left\{e_{0} \otimes e_{\pi(0)}, e_{1} \otimes e_{\pi(1)}, \ldots, e_{d-1} \otimes e_{\pi(d-1)}\right\},
$$

and

$$
\widetilde{\Sigma}_{\alpha}=\left(\mathbb{1} \otimes S^{\alpha}\right) \widetilde{\Sigma}_{0}
$$

It is clear that $\widetilde{\Sigma}_{\alpha}$ and $\widetilde{\Sigma}_{\beta}$ are mutually orthogonal (for $\alpha \neq \beta$ ). Moreover,

$$
\widetilde{\Sigma}_{0} \oplus \ldots \oplus \widetilde{\Sigma}_{d-1}=\mathbb{C}^{d} \otimes \mathbb{C}^{d}
$$

and hence it defines another circulant decomposition. Note, that for $d=2$ one has $\widetilde{\Sigma}_{\alpha}=\Sigma_{\alpha}$. It is no longer true for $d \geq 3$. For $d=3$ one finds

$$
\begin{aligned}
& \widetilde{\Sigma}_{0}=\operatorname{span}\left\{e_{0} \otimes e_{0}, e_{1} \otimes e_{2}, e_{2} \otimes e_{1}\right\}, \\
& \widetilde{\Sigma}_{1}=\operatorname{span}\left\{e_{0} \otimes e_{1}, e_{1} \otimes e_{0}, e_{2} \otimes e_{2}\right\}, \\
& \widetilde{\Sigma}_{2}=\operatorname{span}\left\{e_{0} \otimes e_{2}, e_{1} \otimes e_{1}, e_{2} \otimes e_{0}\right\} .
\end{aligned}
$$

Now, the partially transformed state $\rho^{\tau}$ has again a circulant structure but with respect to the new decomposition (6.13):

$$
\rho^{\Gamma}=\widetilde{\rho}^{(0)}+\ldots+\widetilde{\rho}^{(d-1)}
$$

where

$$
\widetilde{\rho}^{(\alpha)}=\sum_{i, j=0}^{d-1} \widetilde{a}_{i j}^{(\alpha)} e_{i j} \otimes S^{\alpha} e_{\pi(i) \pi(j)} S^{\dagger \alpha}, \quad \alpha=0, \ldots, d-1,
$$

and the new $d \times d$ matrices $\left[\widetilde{a}_{i j}^{(\alpha)}\right]$ are given by the following formulae:

$$
\widetilde{a}^{(\alpha)}=\sum_{\beta=0}^{d-1} a^{(\alpha+\beta)} \circ\left(\Pi S^{\beta}\right), \quad \bmod d,
$$

where "o" denotes the Hadamard product 2 and $\Pi$ being a $d \times d$ permutation matrix corresponding to $\pi$, i.e. $\Pi_{i j}:=\delta_{i, \pi(j)}$. It is therefore clear that our original circulant state (6.8) is PPT iff all $d$ matrices $\widetilde{a}^{(\alpha)}$ satisfy

$$
\widetilde{a}^{(\alpha)} \geq 0, \quad \alpha=0, \ldots, d-1
$$

\subsection{Circulant liftings}

Circulant states provide interesting example of a linear lifting. Denote by $M_{d}$ a $\mathbb{C}^{*}$-algebra of $d \times d$ complex matrices and consider the following lifting

$$
\mathcal{E}^{\#}: S\left(M_{d}\right) \longrightarrow S\left(M_{d} \otimes M_{d}\right)
$$

\footnotetext{
${ }^{2}$ A Hadamard (or Schur) product of two $n \times n$ matrices $A=\left[A_{i j}\right]$ and $B=\left[B_{i j}\right]$ is defined by
}

$$
(A \circ B)_{i j}=A_{i j} B_{i j} .
$$


defined by

$$
\mathcal{E}^{\#}(\rho)=\sum_{\alpha=0}^{d-1} c_{i j}^{(\alpha)} e_{i j} \otimes V_{i \alpha} \rho V_{i \alpha}^{*},
$$

where $\left[c_{i j}^{(\alpha)}\right]$ are $d \times d$ positive matrices for $\alpha=0,1, \ldots, d-1$ such that $\operatorname{Tr} c^{(\alpha)}=1$, and

$$
V_{i \alpha}=\left|e_{i}+e_{\alpha}\right\rangle\left\langle e_{\alpha}\right|
$$

Note that

$$
\mathcal{E}^{\#}(\rho)=\sum_{\alpha=0}^{d-1} a_{i j}^{(\alpha)} e_{i j} \otimes S^{\alpha} e_{i j} S^{\alpha *}
$$

where

$$
a_{i j}^{(\alpha)}=p_{\alpha} c_{i j}^{(\alpha)}
$$

and

$$
p_{\alpha}=\rho_{\alpha \alpha} .
$$

It shows that for any $\rho$ its lifting $\mathcal{E}^{\#}(\rho)$ defines a circulant state. Now, if $c^{(\alpha)}$ are rank-1 projectors, i.e. $c_{m n}^{(\alpha)}=c_{m}^{(\alpha)} \bar{c}_{n}^{(\alpha)}$ are the Grahm matrices for the $d$ complex $d$-vectors $c^{(\alpha)}$, the above formula simplifies to

$$
\mathcal{E}^{\#}(\rho):=V \mathcal{D}(\rho) V^{*}
$$

where

$$
\mathcal{D}(\rho):=\sum_{i=0}^{d-1} e_{i i} \rho e_{i i},
$$

is the projection onto the diagonal part of $\rho$, and

$$
V: \mathbb{C}^{d} \longrightarrow \mathbb{C}^{d} \otimes \mathbb{C}^{d}
$$

is defined by

$$
V e_{\alpha}=\sum_{j=0}^{d-1} c_{j}^{(\alpha)} e_{j} \otimes e_{j+\alpha}
$$

Note, that due the trace condition $\operatorname{Tr} c^{(\alpha)}=1$ the linear operator $V$ defines an isometry

$$
V^{*} V=\mathbb{I}
$$

It should be stressed that the above circulant lifting is never pure.

\subsection{Bell diagonal lifting}

Consider a simplex of Bell diagonal states [25, 26] defined by

$$
\rho=\sum_{m, n=0}^{d-1} p_{m n} P_{m n}
$$


where $p_{m n} \geq 0, \quad \sum_{m, n} p_{m n}=1$ and

$$
P_{m n}=\left(\mathbb{I} \otimes U_{m n}\right) P_{d}^{+}\left(\mathbb{I} \otimes U_{m n}^{\dagger}\right),
$$

with $U_{m n}$ being the collection of $d^{2}$ unitary matrices defined as follows

$$
U_{m n} e_{k}=\lambda^{m k} S^{n} e_{k}=\lambda^{m k} e_{k+n},
$$

with

$$
\lambda=e^{2 \pi i / d} .
$$

The matrices $U_{m n}$ define an orthonormal basis in the space $M_{d}(\mathbb{C})$ of complex $d \times d$ matrices. One easily shows

$$
\operatorname{Tr}\left(U_{m n} U_{r s}^{\dagger}\right)=d \delta_{m r} \delta_{n s} .
$$

It is clear [27] that Bell diagonal states define a subclass of circulant states.

Definition 7 A circulant lifting $\mathcal{E}^{\#}$ is Bell diagonal if $\mathcal{E}^{\#}(\rho)$ defines Bell diagonal state for any $\rho$.

Suppose now that $c^{(0)}=\ldots=c^{(d-1)}=: c$, where the positive matrix $c$ is defined by

$$
c_{k l}=\frac{1}{d} \sum_{m=0}^{d-1} p_{m} \lambda^{m(k-l)} .
$$

The above formula defines a circulant matrix 3

$$
c_{k l}=f_{k-l},
$$

where the $d$-vector $f_{n}$ is defined via the discrete Fourier transform of the probability $d$-vector $p_{m}$. One finds for the lifted state $\rho$

$$
\mathcal{E}^{\#}(\rho)=\sum_{m, n=0}^{d-1} p_{m n}(\rho) P_{m n},
$$

where

$$
p_{m n}(\rho)=p_{m}\langle n|\rho| n\rangle .
$$

Hence, the joint distribution $p_{m n}$ is the product of $p_{m}$ and the classical probability distribution defined by the diagonal elements $\rho_{n n}$ of $\rho$. For more detailed analysis of Bell diagonal states we refer to the recent paper [27].

\footnotetext{
${ }^{3}$ Recall, that a $d \times d$ matrix $c_{i j}$ is circulant if $c_{i j}$ depends only upon the difference $i-j(\bmod d)$. For example

$$
\left(\begin{array}{ll}
a & b \\
b & a
\end{array}\right), \quad\left(\begin{array}{ccc}
a & b & c \\
c & a & b \\
b & c & a
\end{array}\right)
$$
}

are circulant matrices. 


\section{Conclusions}

We analyzed the procedure of lifting in classical stochastic and quantum systems. Lifting enables one to 'lift' a state of a classical/quantum system into a state of 'system+reservoir'. This procedure is important both in quantum information theory and in the theory of open systems. It turns out that it is very much related to the problem of completely positive maps are the workhorse in these fields. We discussed both linear and nonlinear liftings and provided instructive illustration of the general theory by a particular class related to so called circulant states. In particular it is shown how to lift a state of a subsystem to the Bell diagonal state of the composed system. The theory of liftings may provide new constructions of classical/quantum channels. Moreover, it may be used to construct new classes of linear maps which are positive but not completely positive. It is well known that such maps define a basic tool for detection quantum entanglement. We therefore conclude that the theory of liftings might provide an interesting insight both in quantum information theory and in the intricate mathematics of (completely) positive maps. Both problems deserve further studies.

\section{Acknowledgement}

DC and AK were supported by the Polish Ministry of Science and Higher Education Grant No 3004/B/H03/2007/33. TM was supported by the QBIC grant.

\section{References}

[1] M. A. Nielsen and I. L. Chuang, Quantum Computation and Quantum Information, (Cambridge University Press, Cambridge, England, 2000).

[2] R. Horodecki, P. Horodecki, M. Horodecki and K. Horodecki, Quantum Entanglement, Rev. Mod. Phys. 81, 865 (2009).

[3] L. Accardi and M. Ohya, Compound Channels, Transition Expectations, and Liftings, Appl. Math. Opt. 39 (1999) 33-59.

[4] V. P. Belavkin and M. Ohya, Entanglements and compound states in quantum information theory, arXiv:quant-ph/0004069.

[5] V. P. Belavkin and M. Ohya, Entanglement, quantum entropy and mutual information, Proc. R. Soc. Lond. A 458 (2002) 209-231.

[6] J. Kupsch, O. G. Smolyanov, N. A. Sidorova, States of quantum systems and their liftings, J. Math. Phys. 42 (2001) 1026.

[7] R. Alicki and K. Lendi, Quantum Dynamical Semigroups and Applications (Springer, Berlin, 1987).

[8] H.-P. Breuer and F. Petruccione, The Theory of Open Quantum Systems (Oxford Univ. Press, Oxford, 2007). 
[9] P. Pechukas, Reduced Dynamics Need Not Be Completely Positive, Phys. Rev. Lett. 73, 1060 (1994)

[10] R. Alicki, Comment on Reduced Dynamics Need Not Be Completely Positive, Phys. Rev. Lett. 75, 3020 (1995).

[11] A. Shaji and E. C. G. Sudarshan, Who's affraid of not completely positive maps?, Phys. Lett. A 341, 48-54 (2005).

[12] C. Rodriguez, K. Modi, A. Kuah, E. C. G. Sudarshan, and A. Shaji, Completely positive maps and classical correlations, J. Phys. A 41, 205301 (2008).

[13] A. Shabani and D. Lidar, Vanishing quantum discord is necessary and sufficient for completely positive maps, Phys. Rev. Lett. 102, 100402 (2009).

[14] H. Carteret, D.R. Terno and K. Życzkowski, Dynamics beyond completely positive maps: some properties and applications, Phys. Rev A 77, 042113 (2008).

[15] D. Chruściński and A. Kossakowski, Non-Markovian quantum dynamics: local versus nonlocal, Phys. Rev. Lett. 104, 070406 (2010).

[16] D. Chruściński, A. Kossakowski and S. Pascazio, Long-time memory in non-Markovian evolution, Phys. Rev. A 81, 032101 (2010).

[17] D. Chruściński, A. Kossakowski, On the structure of entanglement witnesses and new class of positive indecomposable maps, Open Systems and Inf. Dynamics, 14, 275-294 (2007).

[18] D. Chruściński and A. Kossakowski, A class of positive atomic maps, J. Phys. A: Math. Theor. 41, (2008) 215201.

[19] D. Chruściński and A. Kossakowski, Geometry of quantum states: new construction of positive maps, Phys. Lett. A 373, (2009) 2301-2305.

[20] D. Chruściński and A. Kossakowski, Spectral properties of positive maps, Comm. Math. Phys. 290, 1051 (2009).

[21] D. Chruściński, J. Pytel and G. Sarbicki, Constructing optimal entanglement witnesses, Phys. Rev. A 80 (2009) 062314.

[22] D. Chruściński, A. Kossakowski and G. Sarbicki, Spectral conditions for entanglement witnesses versus bound entanglement, Phys. Rev. A 80 (2009) 042314.

[23] D. Chruściński and A. Kossakowski, On circulant states with positive partial transpose, Phys. Rev. A 76, 032308 (2007).

[24] D. Chruściński and A. Pittenger, Generalized Circulant Densities and a Sufficient Condition for Separability, J. Phys. A: Math. Theor. 41, 385301 (2008).

[25] R. A. Bertlmann, and Ph. Krammer, Bound entanglement in the set of Bell-state mixtures of two qutrits, Phys. Rev. A 78, 014303 (2008). 
[26] B. Baumgartner, B. Hiesmayr, and H. Narnhofer, A special simplex in the state space for entangled qudits, J. Phys. A: Math. Theor. 40, 7919 (2007).

[27] D. Chruściński, A. Kossakowski, K. Młodawski and T. Matsuoka, A class of Bell diagonal states and entanglement witnesses, Open Systems and Inf. Dynamics 17, 235-254 (2010).

[28] C. Cecchini, D. Petz, Classes of conditional expectations over von Neumann algebras, J. Func. Anal. 91 (1990) 8-29.

[29] C. Cecchini, D. Petz, State extention and a Radon-Nikodym theorem for conditional expectations on von Nuemann algebras, Pacific Journal of Mathematics, 138 (1989) 9-24.

[30] L. Accardi, T. Matsuoka and M. Ohya, Entangled Markow Chains are Indeed Entangled, Infin. Dim. Anal. Quantum Probab. Relat. Top. 9 (2006) 379-390.

[31] V. Scarani, S. Iblisdir, N. Gisin and A. Acin, Quantum cloning, Rev. Mod. Phys. 77, 1225-1256 (2005).

[32] G. M. D'Ariano, C. Macchiavello and P. Perinotti, Superbroadcasting of Mixed States, Phys. Rev. Lett. 95, (2005) 060503.

[33] A. Kossakowski, M. Ohya and N. Watanabe, Quantum Dynamical Entropy for Completely Positive Maps, Infin. Dim. Anal. Quantum Probab. Relat. Top. 2, (1999) 267-282.

[34] M. Asorey, A. Kossakowski, G. Marmo, E.C.G. Sudarshan, Relations Between Quantum Maps and Quantum States, Open. Syst. Info. Dyn. 12, (2006) 319-329.

[35] A. Cerf and C. Adami, Negative entropy and information in quantum mechanics, Phys. Rev. Lett. 79 (1997) 5194.

[36] L. Accardi, Topics in quantum probability, Phys. Rep. 77 (1981) 169-192. 